\title{
Estimación de producción primaria, biomasa y productividad fitoplanctónica y su relación con algunas condiciones ambientales en la laguna "Paraíso", Huacho - 2016
}

Estimation of primary production, biomass and phytoplankton productivity and its relationship with some environmental conditions in the "Paraíso" lagoon, Huacho - 2016

Hermila Belba Díaz Pillasca', Francisco Castillo More', Zoila Felipa Honorio Durand², Miguel Angel Durand ${ }^{1}$

\section{RESUMEN}

Objetivo: Cuantificar la producción primaria, índice de productividad y biomasa del fitoplancton; y estimar su relación con algunas condiciones ambientales del cuerpo de agua. Métodos: Descriptivo - correlacional, de una sola casilla; con $n=120$ de una población que tiende al infinito; 90 elegidas con muestreo aleatorio estratificado (30 por estrato); y 30 con muestreo aleatorio sistemático. Los datos se analizaron mediante estadística descriptiva e inferencial $(\alpha=0,05)$. Resultados: Se encontró heterogeneidad de los datos entre pigmentos y puntos de muestreo (estratos), en todas las variables e indicadores; reflejada en los promedios (todos los casos); ratificada con las medidas de dispersión; y, confirmada por el análisis de varianza; donde la Prueba de Tukey indicó que los valores mayores se dieron en el estrato 3 (oxígeno), estrato 1 (Productividad Total) y estrato 2 (Productividad Primaria Neta e Índice de Productividad); y en pigmentos, la clorofila "a" fue la más abundante. La Temperatura del aire mostró una relación negativa con el Índice de Productividad y positiva con la biomasa; la misma que se relacionó con la Transparencia, Coeficiente de extinción y Punto de compensación. Conclusiones: La Productividad Primaria Neta es buena; ratificado por el alto valor del Índice de Productividad; y reafirmado por la tasa de biomasa fitoplactónica. La temperatura del agua influye negativamente en la productividad total, primaria neta, índice de productividad y en la biomasa.

Palabras clave: Humedal, productividad, biomasa, fitoplancton.

\section{ABSTRACT}

Objective: Quantify the primary production, productivity index and phytoplankton biomass; and estimate its relationship with some environmental conditions of the body of water. Methods: Descriptive - correlational, single-cell; with $n=120$ of a population that tends to infinity; 90 chosen with stratified random sampling (30 per stratum); and 30 with systematic random sampling. The data were analyzed by descriptive and inferential statistics $(\alpha=0.05)$. Results: We found heterogeneity of the data between pigments and sampling points (strata), in all the variables and indicators; reflected in the averages (all cases); ratified with dispersion measures; and, confirmed by the analysis of variance; where the Tukey test indicated that the highest values were given in stratum 3 (oxygen), stratum 1 (Total Productivity) and stratum 2 (Net Primary Productivity and Productivity Index); and in pigments, chlorophyll "a" was the most abundant. The Air Temperature showed a negative relationship with the Productivity Index and positive with the biomass; the same as that related to Transparency, Extinction Coefficient and Compensation Point. Conclusions: The Net Primary Productivity is good; ratified by the high value of the Productivity Index; and reaffirmed by the phytoplankton biomass rate. Water temperature negatively affects total productivity, primary net, productivity index and biomass.

Key words: Wetland, productivity, biomass, phytoplankton.

\footnotetext{
1 Facultad de Ciencias, Universidad Nacional José Faustino Sánchez Carrión. Huacho, Perú.

${ }^{2}$ Facultad de Bromatología y Nutrición. Universidad Nacional José Faustino Sánchez Carrión. Huacho, Perú.
} 


\section{INTRODUCCIÓN}

La importancia de los humedales naturales ha sido reiteradamente indicada (Ramsar, 2018) por las funciones medio ambientales que realizan; al controlar los cursos de las corrientes de agua, participar en la regulación del carbono global, proporcionar hábitat a centenares de especies animales y vegetales, y poseer un importante valor cultural y recreacional (Clarkson et al., 2004; Rodríguez, 2008). Más recientemente se ha comenzado a estudiar su rol a escala global en la productividad primaria, mediante los ciclos del oxígeno, carbono, azufre y nitrógeno a través de la biosfera; en particular, interesa su dinámica en cuanto al metabolismo del carbono, o su contraparte, en el contexto de los estudios de cambio global (Fuam, 2013).

Algunos humedales poseen grandes cantidades de materia orgánica proveniente de las macrófitas y el plancton presentes en ellos; los cuales pueden influir en la estructura de las redes tróficas planctónicas y el metabolismo general de todo el cuerpo de agua (Rodríguez, 2008); al actuar como fuente de carbono, promoviendo el crecimiento del bacterioplancton, que pasa a competir con el fitoplancton por los nutrientes inorgánicos, disminuyendo la producción primaria de los humedales (Falkowski y Raven, 2007).

El fitoplancton, es el componente biótico más importante de los humedales, e influenciado por un conjunto de factores, bióticos y abióticos, que limitan o regulan la producción primaria; siendo indispensable su evaluación, porque las funciones, los valores y atributos en cuestión sólo pueden mantenerse si se los conoce y se permite que los procesos ecológicos sigan funcionando A pesar de los avances logrados, estos ecosistemas son los más amenazados del mundo, por la desecación, contaminación y sobreexplotación Gil, et al., (2013) de allí que, a nivel internacional, son numerosos los estudios orientados a conocer algún aspecto de los humedales, con el fin de lograr su conservación y uso racional; como ejemplo, basta con mencionar dos de ellos, la evaluación del biovolumen fitoplanctónico de lagos y lagunas del país Vasco (Fuam, 2013) $\mathrm{y}$, estructura y producción primaria del fitoplancton y perifiton en un humedal del bajo Paraná (Rodriguez, 2008).

En Perú, se han estudiado diversas comunidades acuáticas continentales de costa, sierra y selva, en aspectos de taxonomía, distribución y ecología de la biodiversidad faunística, florística y fitoplanctónica; así como la caracterización físico-química del agua (Wilhem, 1981; Ortega et al. (2014) Existen muchos más autores; todos con el mismo enfoque de los ya mencionados; más no hay trabajos orientados a evaluar la fisiología de estos ecosistemas. Situación semejante que se presenta en la laguna Paraíso (Huacho), de la cual el único trabajo que existe es el inventario florístico realizado por Cano et al. (1998). Por ello, el presente trabajo estuvo orientado a cuantificar la producción primaria, índice de productividad y biomasa del fitoplancton; y, estimar su relación con las condiciones ambientales del cuerpo de agua como temperatura, $\mathrm{pH}$, Oxígeno disuelto, coeficiente de extinción de la luz $(K)$, profundidad y transparencia. La evaluación de la producción primaria, biomasa y productividad del fitoplancton de la laguna Paraíso, se justifica plenamente por la trascendencia que tienen los humedales desde el punto de vista ecológico, científico, económico, turístico y paisajístico.

\section{MATERIAL Y MÉTODOS}

El tipo de estudio fue descriptivo correlacional; con diseño de investigación de una sola casilla; donde la población tiende al infinito, al estar constituida por la medición repetida de las unidades muestrales; siendo 120 el tamaño de muestra, estimada con la fórmula $n=\left[Z^{2} P . Q\right] /[E E]^{2} ; \operatorname{con} \alpha=0,05 ; P=Q=$ 0,$5 ; y, E E=0,0895$; elegidas con muestreo aleatorio estratificado; con tres estratos (puntos de muestreo), extrayéndose de cada uno 30 muestras para estimar producción primaria y productividad; y para la biomasa, 
las 30 muestras se obtuvieron con muestreo aleatorio sistemático.

Para estimar la producción primaria se aplicó el método de las botellas claras y oscuras (Gaarder y Gran, 1927), mientras que la biomasa se evaluó con el método tricromático de cuantificación de pigmentos fotosintetizadores por espectrofotometría, aplicando las fórmulas de Jeffrey y Humphrey (1975); empleándose la fórmula propuesta por Brower et al. (1990) para estimar la productividad.

Como indicadores ambientales, se midió la temperatura de aire y agua con un termómetro convencional y de cubeta, respectivamente; el $\mathrm{pH}$ fue medido con un potenciómetro digital; para estimar el oxígeno disuelto se aplicó el método de Winkler, descrito en Parsson, Maita y Lalli (1984); el coeficiente de extinción (K) se estimó mediante la fórmula de Poole y Atkins descrito en Gras Olivares (1991); empleándose una sonda graduada para medir la profundidad del cuerpo de agua; y, su transparencia se expresó como la profundidad de visión del disco de Secchi.

Los datos fueron analizados mediante estadística descriptiva (media y dispersión) y estadística inferencial; a través de análisis de varianza para estimar diferencias por variable e indicadores entre puntos de muestreo y entre pigmentos; y la Prueba de Tukey permitió tipificar las diferencias. La relación de indicadores con las variables evaluadas se estimó con el coeficiente de correlación lineal de Pearson; probándose las diferencias, en todos los casos, con un nivel de confianza de $95 \%$.

\section{RESULTADOS}

Se describen, estadísticamente, las variables e indicadores (Tabla 1). Los resultados muestran heterogeneidad de los datos dentro y entre pigmentos, así como en los puntos de muestreos, en todas las variables e indicadores; de allí la heterogeneidad de promedios, en todos los casos; ratificada con las medidas de dispersión; teniendo la clorofila "c" la mayor variabilidad (CV=64,23\%).

El análisis de varianza (Tabla 2), con $\alpha=0,05$, indica que, en todos los casos, por lo menos un factor es diferente a los demás, dentro de cada variable. La discriminación de las diferencias mediante la Prueba de Tukey (Tabla 3), indica que, en promedio, el tenor de oxígeno en el punto de muestreo 03 (11,95 $\mathrm{mg} / \mathrm{L}$ ) es mayor $y$ diferente al valor correspondiente a los otros puntos de muestreo y total, los cuales son iguales entre sí.

La productividad total en el punto de muestreo 01 (555,00 $\left.\mathrm{mg} \mathrm{C} / \mathrm{m}^{3} / \mathrm{h}\right)$, es mayor y diferente a los demás; y la productividad primaria neta del punto de muestreo $03\left(119,58 \mathrm{mg} \mathrm{C} / \mathrm{m}^{3} / \mathrm{h}\right)$, es menor y diferente a los otros. Los promedios del índice de productividad (IP) para el punto de muestreo $02(72,97 \%)$ y promedio total $(70,668 \%)$, son iguales entre sí y diferentes a los demás; siendo el valor más bajo el correspondiente al punto de muestreo 03 $(47,56 \%)$ y diferente al promedio de punto de muestreo 01 (54,08\%).

Además, se confirma que la clorofila "a" $\left(1,7979 \mathrm{mg} / \mathrm{m}^{3}\right)$ es más abundante, seguida de los carotenos; teniendo los demás pigmentos una diferenciación gradual; siendo menos abundantes e iguales entre sí los feopigmentos y clorofila "b". 
Tabla 1. Valores de media, error estándar (EE), desviación estándar (DE), y coeficiente de variación (CV) en estratos; oxígeno ( $\mathrm{mg} / \mathrm{L})$, productividad $\left(\mathrm{mgC} / \mathrm{m}^{3} /\right.$ hora), y en total de la laguna: biomasa $\left(\mathrm{mg} / \mathrm{m}^{3}\right)$ e indicadores ambientales, de la laguna Paraíso - Huacho, 2016.

\begin{tabular}{|c|c|c|c|c|c|c|c|}
\hline & Estratos & Variables e indicadores & $\mathbf{n}$ & Media & EE & DE & CV \\
\hline \multirow{12}{*}{ 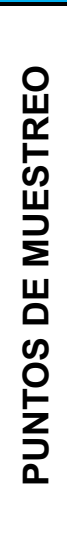 } & \multirow{5}{*}{1} & Oxígeno & 30 & 8,837 & 0,255 & 1,398 & 15,82 \\
\hline & & Productividad Primaria Neta & 30 & 297,42 & 3,97 & 21,73 & 7,3 \\
\hline & & Productividad Total & 30 & 555 & 10,3 & 56,6 & 10,2 \\
\hline & & Índice de Productividad & 30 & 54,08 & 1,15 & 6,29 & 11,63 \\
\hline & & Oxígeno & 30 & 9,195 & 0,42 & 2,302 & 25,04 \\
\hline & \multirow{4}{*}{2} & Productividad Primaria Neta & 30 & 323,8 & 11,6 & 63,3 & 19,57 \\
\hline & & Productividad Total & 30 & 446,3 & 16,4 & 89,6 & 20,08 \\
\hline & & Índice de Productividad & 30 & 72,97 & 1,37 & 7,48 & 10,25 \\
\hline & & Oxígeno & 30 & 11,995 & 0,513 & 2,81 & 23,43 \\
\hline & \multirow{3}{*}{3} & Productividad Primaria Neta & 30 & 119,58 & 7,6 & 41,61 & 34,8 \\
\hline & & Productividad Total & 30 & 261,3 & 16 & 87,6 & 33,54 \\
\hline & & Índice de Productividad & 30 & 47,56 & 2,22 & 12,14 & 25,53 \\
\hline \multirow{16}{*}{ 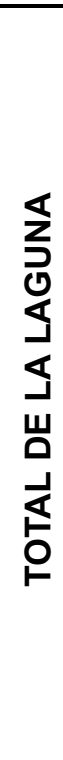 } & \multicolumn{2}{|c|}{ Productividad Primaria Neta } & 30 & 297,42 & 3,97 & 21,73 & 7,3 \\
\hline & \multicolumn{2}{|c|}{ Productividad Total } & 30 & 420,91 & 4,37 & 23,95 & 5,69 \\
\hline & \multicolumn{2}{|c|}{ Índice de Productividad } & 30 & 70,668 & 0,603 & 3,3 & 4,67 \\
\hline & \multicolumn{2}{|c|}{ Pigmentos (Biomasa) } & 30 & 4,815 & 0,073 & 0,398 & 8,26 \\
\hline & \multirow{7}{*}{ 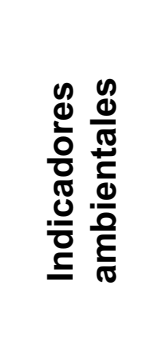 } & Oxígeno & 30 & 10,009 & 0,395 & 2,162 & 21,6 \\
\hline & & $\mathrm{pH}$ & 30 & 7,033 & 0,08 & 0,441 & 6,26 \\
\hline & & Temperatura del aire & 30 & 31,567 & 0,133 & 0,728 & 2,31 \\
\hline & & Temperatura del agua & 30 & 29,933 & 0,239 & 1,311 & 4,38 \\
\hline & & $\begin{array}{l}\text { Profundidad (Transparencia } \\
=\mathrm{T} \text { ) }\end{array}$ & 30 & 0,292 & 0,008 & 0,043 & 14,67 \\
\hline & & Coeficiente de extinción $\mathrm{K}$ & 30 & 5,946 & 0,17 & 0,931 & 15,66 \\
\hline & & Punto de compensación Pc & 30 & 0,789 & 0,021 & 0,117 & 14,77 \\
\hline & \multirow{5}{*}{ 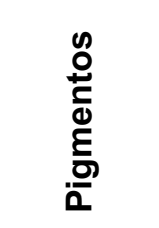 } & Clorofila "a" & 30 & 1,798 & 0,012 & 0,063 & 3,51 \\
\hline & & Clorofila "b" & 30 & 0,419 & 0,021 & 0,114 & 27,22 \\
\hline & & Clorofila "c" & 30 & 0,556 & 0,065 & 0,357 & 64,23 \\
\hline & & Feopigmentos & 30 & 0,532 & 0,018 & 0,096 & 18,06 \\
\hline & & Carotenos & 30 & 1,51 & 0,005 & 0,029 & 1,9 \\
\hline
\end{tabular}

Tabla 2. Análisis de varianza para $\mathrm{O}_{2}$, Productividad e Índice de Productividad entre puntos de muestreo; y, entre pigmentos de la laguna El Paraíso - Huacho, 2016.

\begin{tabular}{|c|c|c|c|c|c|c|}
\hline Variables/Indicadores & Fuentes & gl & $\begin{array}{c}\text { Suma de } \\
\text { Cuadrados }\end{array}$ & $\begin{array}{l}\text { Cuadrado } \\
\text { medio }\end{array}$ & “F” & “P” \\
\hline \multirow{3}{*}{ Oxígeno $\left(\mathrm{O}_{2}\right)$} & Factor & 3 & 179,4 & 59,796 & \multirow{3}{*}{12,07} & \multirow{3}{*}{0} \\
\hline & Error & 116 & 574,9 & 4,956 & & \\
\hline & Total & 119 & 754,3 & & & \\
\hline \multirow{3}{*}{ Productividad (P) } & Factor & 7 & 3682066 & 526009 & \multirow{3}{*}{160,8} & \multirow{3}{*}{0} \\
\hline & Error & 232 & 759053 & 3272 & & \\
\hline & Total & 239 & 4441118 & & & \\
\hline \multirow{3}{*}{ Índice de Productividad (IP) } & Factor & 3 & 13938 & 4646,15 & \multirow{3}{*}{73,23} & \multirow{3}{*}{0} \\
\hline & Error & 116 & 7360 & 63,45 & & \\
\hline & Total & 119 & 21298 & & & \\
\hline \multirow{3}{*}{ Pigmentos } & Factor & 4 & 49,287 & 12,3218 & \multirow{3}{*}{396,2} & \multirow{3}{*}{0} \\
\hline & Error & 145 & 4,487 & 0,0309 & & \\
\hline & Total & 149 & 53,775 & & & \\
\hline
\end{tabular}


Tabla 3. Prueba de Tukey para observar diferencias de promedios.

\begin{tabular}{|c|c|c|c|c|}
\hline & & $\mathrm{n}$ & Media & Agrupación \\
\hline \multirow{4}{*}{ OXÍGENO } & Puesto de muestreo 03 (PM 03) & 30 & 11,995 & $A$ \\
\hline & Promedio Total & 30 & 10,009 & B \\
\hline & Puesto de muestreo 02 (PM 02) & 30 & 9,195 & B \\
\hline & Puesto de muestreo 01 (PM 01) & 30 & 8,837 & B \\
\hline \multirow{8}{*}{ 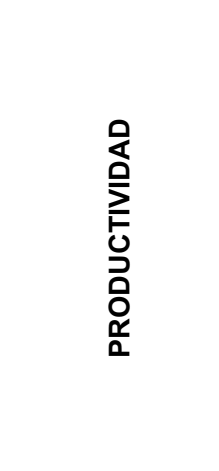 } & Productividad Total de PM 01 & 30 & 555 & $A$ \\
\hline & Productividad Total de PM 02 & 30 & 446,3 & B \\
\hline & $\begin{array}{l}\text { Productividad Total de Promedio } \\
\text { Global }\end{array}$ & 30 & 420,91 & B \\
\hline & & 30 & 323,8 & $C$ \\
\hline & $\begin{array}{l}\text { Productividad Primaria Neta de PM } \\
01\end{array}$ & 30 & 297,42 & $\mathrm{D}$ \\
\hline & Productividad Primaria Neta Global & 30 & 297,42 & C \\
\hline & Productividad Total de PM 03 & 30 & 261,3 & c \\
\hline & $\begin{array}{l}\text { Productividad Primaria Neta de PM } \\
03\end{array}$ & 30 & 119,58 & $E$ \\
\hline \multirow{4}{*}{ ÍNDICE DE “P” } & Índice de Productividad de PM 02 & 30 & 72,97 & A \\
\hline & Índice de Productividad Total & 30 & 70,668 & $A$ \\
\hline & Índice de Productividad de PM 01 & 30 & 54,08 & B \\
\hline & Índice de Productividad de PM 03 & 30 & 47,56 & C \\
\hline \multirow{5}{*}{ PIGMENTOS } & Clorofila "a" & 30 & 1,7979 & $A$ \\
\hline & Carotenos & 30 & 1,5097 & B \\
\hline & Clorofila "c" & 30 & 0,5563 & C \\
\hline & Feopigmentos & 30 & 0,5323 & $C D$ \\
\hline & Clorofila "b" & 30 & 0,419 & $\mathrm{D}$ \\
\hline
\end{tabular}

Las medias que no comparten una letra son significativamente diferentes.

Los valores de correlación (Tabla 4), indican que, estadísticamente a un nivel de confianza del $95 \%$, la temperatura del aire tiene relación negativa con el IP $(r=-0,42)$ y positiva con la biomasa $(r=0,41)$. Así mismo, los indicadores profundidad (transparencia), K y Pc están correlacionados con la biomasa, siendo negativa la relación con $\mathrm{K}(-0,428)$.

Tabla 4. Valores de correlación $(r)$ y "p" $(\alpha=0,05)$ de productividad y biomasa con indicadores de la variable ambiental, laguna El Paraíso - Huacho, 2016.

\begin{tabular}{|c|c|c|c|c|c|c|}
\hline & Indicadores & & $\begin{array}{l}\text { Productividad } \\
\text { Primaria Neta }\end{array}$ & Total & IP & Biomasa \\
\hline \multirow{14}{*}{ 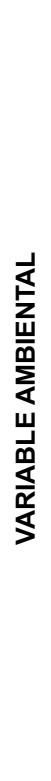 } & \multirow{2}{*}{ Oxígeno $\left(\mathrm{O}_{2}\right)$} & $r$ & 0,046 & 0,1 & $-0,043$ & 0,243 \\
\hline & & $\mathrm{p}$ & 0,811 & 0,598 & 0,821 & 0,196 \\
\hline & \multirow{2}{*}{$\begin{array}{l}\text { Potencial de } \\
\text { Hidrogeniones }(\mathrm{pH})\end{array}$} & $r$ & $-0,061$ & $-0,227$ & 0,189 & $-0,228$ \\
\hline & & $\mathrm{p}$ & 0,747 & 0,228 & 0,316 & 0,225 \\
\hline & \multirow{2}{*}{$\begin{array}{l}\text { Temperatura del aire } \\
\text { (Taire) }\end{array}$} & $r$ & $-0,039$ & 0,278 & $-0,42$ & 0,41 \\
\hline & & $\mathrm{p}$ & 0,838 & 0,137 & 0,021 & 0,024 \\
\hline & \multirow{2}{*}{$\begin{array}{l}\text { Temperatura del agua } \\
\text { (Tagua) }\end{array}$} & $r$ & $-0,253$ & $-0,055$ & $-0,345$ & 0,236 \\
\hline & & $\mathrm{p}$ & 0,177 & 0,772 & 0,062 & 0,209 \\
\hline & \multirow{2}{*}{$\begin{array}{l}\text { Profundidad } \\
\text { (Transparencia) }\end{array}$} & $r$ & 0,165 & 0,158 & 0,048 & 0,435 \\
\hline & & $\mathrm{p}$ & 0,382 & 0,405 & 0,8 & 0,016 \\
\hline & \multirow{2}{*}{$\begin{array}{l}\text { Coeficiente de extinción } \\
\text { de la luz }(K)\end{array}$} & $r$ & $-0,164$ & $-0,14$ & $-0,066$ & $-0,428$ \\
\hline & & $\mathrm{p}$ & 0,387 & 0,46 & 0,728 & 0,018 \\
\hline & \multirow{2}{*}{$\begin{array}{l}\text { Punto de compensación } \\
\text { (Pc) }\end{array}$} & $r$ & 0,166 & 0,15 & 0,06 & 0,434 \\
\hline & & $\mathrm{p}$ & 0,38 & 0,43 & 0,754 & 0,016 \\
\hline
\end{tabular}




\section{DISCUSIÓN}

La Laguna Paraíso es un cuerpo de agua somero, alimentada por los acuíferos que en ella afloran; $y$, donde el viento ejerce un importante papel en la mezcla constante de sus aguas. Su ambiente lumínico es restringido en cuanto a la cantidad y calidad de luz que penetra, debido principalmente a las altas concentraciones de sustancias orgánicas en descomposición; atenuando la irradiancia en sí y proveyendo una importante fuente autóctona de ácidos húmicos como lo señala Wilhem (1981). Estas restricciones sin embargo no minimizan el desarrollo fitoplanctónico por lo que, la laguna se encuentra en un estado de aguas turbias; $y$, presumiblemente con características mixotróficas como lo explican Gil et al. (2013).

Por otro lado, las concentraciones de oxígeno disuelto en un cuerpo léntico, como es el caso de la Laguna Paraíso, están condicionadas por una serie de variables ambientales; siendo algunas de ellas el viento, la temperatura, $\mathrm{pH}$, transparencia; así como la abundancia relativa de organismos fotosintetizadores y consumidores, explicados por Brower et al. (1990). Además, las características de éstas y otras variables pueden cambiar durante las horas del día y del espacio ocupado por el espejo de agua, lo cual repercutirá en las estimaciones de la concentración de oxígeno disuelto en el cuerpo de agua (Fuman, 2013).

De allí que, en directa relación con lo afirmado en el párrafo anterior, los resultados obtenidos para los valores de oxígeno disuelto en la Laguna Paraíso (Tabla 1), muestran tendencia a la heterogeneidad dentro y entre los puntos de muestreos evaluados; tal como lo confirman los valores del coeficiente de variación (CV). Además, el tenor promedio de oxígeno disuelto para la laguna, indica que tal concentración se ubica dentro de la categoría de bueno como lo califica Rodríguez (2008).

Considerando que el método de Winkler, aplicado para medir el oxígeno, y en base a la estequiometria de la ecuación general de la fotosíntesis, permite estimar las tasas de fotosíntesis bruta, neta y de respiración; expresadas como producción de oxígeno o consumo de $\mathrm{CO}_{2}$; y, a partir de estos valores, se puede calcular la productividad (Parssonm Maita y Lalli, 1084), y que la fotosíntesis al proveer de materia orgánica primaria como fuente de energía química para los demás organismos del ecosistema; pone un límite superior sobre la biomasa total y productividad de los ecosistemas; y, restringe el flujo de energía sobre la superficie de los mismos como lo indican Falkowski y Raven (2007), por lo que se esperaba que los valores estimados de oxígeno disuelto ( $\mathrm{mg} / \mathrm{L})$, dentro y entre puntos de muestro y promedio total, tengan una estrecha relación con las tasas de fotosíntesis (bruta y neta) y respiración; y, por ende, con la productividad total (bruta) y neta del ecosistema. Sin embargo, los resultados indican una ausencia total de esta relación (Tabla 4).

Estos resultados pueden ser debidos a diversas causas; siendo la principal, el método empleado; puesto que la respiración que se mide en la botella oscura, no es sólo de las algas sino de todos los componentes del plancton y asume, además, que la respiración en la botella clara es equivalente a la que ocurre en la botella oscura. Este supuesto no siempre es válido ya que en la oscuridad puede existir proliferación bacteriana cuya respiración afectaría una estimación precisa de la respiración algal como fue señalado por Clarkson et al. (2004).

Esta afirmación, es confirmada por lo observado en la laguna; ya que, en diferentes puntos del cuerpo de agua, entre la vegetación emergente, descansan y anidan poblaciones de Phalacrocorax brasilianus "cuervo de mar"; cuyas deyecciones son incorporadas al agua, incrementando la población bacteriana, las cuales modifican las tasas de respiración y enmascaran el tenor real de la respiración del fitoplancton.

La presencia de sustancias orgánicas en descomposición favorece el crecimiento de la población bacteriana, al tiempo que disminuye la productividad primaria neta de las algas. Además, la distribución de estas sustancias, y por ende de los microrganismos desintegradores, no es uniforme en el cuerpo de agua; reflejándose en la heterogeneidad de los datos de las variables e indicadores evaluados; lo que explica, por ejemplo, la tasa de productividad primaria neta más baja $\left(119,85 \mathrm{mg} \mathrm{C} / \mathrm{m}^{3} / \mathrm{h}\right)$ en el punto de muestreo 03 (Tabla 1); porque es la zona donde hay una mayor población de cuervos de mar. A pesar de ello, en promedio, la productividad primaria 
neta de la laguna $\left(297,42 \mathrm{mg} \mathrm{C} / \mathrm{m}^{3} / \mathrm{h}\right)$, puede calificarse de buena, correspondiendo a un ecosistema euproductivo ( $>250 \mathrm{mgC} / \mathrm{m}^{3} / \mathrm{h}$ ) como fue reportado por Gil et al. (2013).

Por otro lado, se debe considerar que la productividad total (bruta) mide la tasa de fijación de carbono sin considerar las pérdidas respiratorias, que sí se tienen en cuenta cuando se evalúa la productividad primaria neta; por tanto, un mejor indicador de la eficiencia de productividad del ecosistema es el Índice de Productividad señalado por Falkowski y Raven (2007), el mismo que en el presente trabajo, muestra que el IP es menor del $50 \%$ en el punto de muestreo 03 (Tabla 1), lo cual ratifica lo afirmado en párrafos anteriores.

En cuanto a la abundancia relativa de los pigmentos (Tabla 1) que contribuyen con la biomasa algal, predominan la clorofila "a", seguida de los carotenos; ocupando el último lugar la clorofila "b"; lo cual es fácilmente explicable; porque en todos los organismos fitoplanctónicos están presentes estos pigmentos; mientras que los demás, sólo predominan en algunos grupos taxonómicos y no en otros (Falkowski y Raven, 2007)

La estimación de la biomasa fitoplanctónica es una de las actividades de mayor interés, para una adecuada gestión ambiental de las masas de agua continentales (Fuaman, 2013), sin embargo, la estimación de esta importante variable ecológica resulta siempre controvertida por encontrarse sujeta a diferentes aproximaciones metodológicas, relacionadas con el nivel de esfuerzo y costo económico (Ortega et al., 2014). Aun así, la mayoría de autores, recomiendan la estimación volumétrica de biomasa expresada en $\mathrm{mg} / \mathrm{m}^{3}$, que es el método aplicado en el presente trabajo. Bajo este criterio, se puede afirmar que el valor promedio de biomasa es bueno; y corresponde a un ecosistema euproductivo (Fuam, 2013).

De los diversos factores que determinan las condiciones ecológicas de un cuerpo léntico, como es el caso de la laguna EI Paraíso; es tal vez la temperatura, el que tiene mayor influencia; pues determina la densidad, viscosidad y movimiento del agua, debido a las propiedades térmicas de ésta; repercutiendo en la distribución, periodicidad y reproducción de los organismos como lo explica Nixon (1981). La variación de la temperatura puede deberse a diversas causas; sin embargo, las principales son la ubicación geográfica del cuerpo de agua y la estación del año. De allí que, los resultados encontrados para la temperatura del aire y del agua, en la laguna evaluada, concuerdan con los rangos señalados para humedales costeros de Gil et al. (2013).

Así mismo, las condiciones ópticas de las aguas son de importancia primordial para la productividad biológica y para el mantenimiento de la vida; siendo la transparencia del agua y sus variables sintéticas (coeficiente de extinción de la luz y profundidad de compensación), las que afectan la penetración de la luz; debido a la presencia de sustancias orgánicas en descomposición, sólidos en suspensión y concentración planctónica; característica común de los humedales; por ello, de acuerdo con los resultados obtenidos en el presente trabajo $(T=0,292 \mathrm{~m}, \mathrm{~K}=5,946 \mathrm{~m} ; \mathrm{Pc}=$ $0,789 \mathrm{~m})$, se puede afirmar que la laguna El Paraíso, tiene un estado eutrófico; razón por la cual, la biomasa fitoplanctónica indica que se trata de un ecosistema euproductivo; asociado a la intensidad lumínica; es por ello que, la transparencia y sus variables sintéticas están asociadas al índice de productividad y biomasa; ratificadas, indirectamente, por la relación positiva de la temperatura del aire con la biomasa y negativamente con el índice de productividad (Tabla 4) coincidiendo con lo escrito por Brower et al. (1990) y Clarkson, et al. (2004).

En cuanto al valor del $\mathrm{pH}$ promedio registrado en este cuerpo de agua $(\mathrm{pH}=7,033)$, se ubica muy cercano a la neutralidad; es decir se encuentra dentro del rango $(6,5$ y 8,7$)$ reportado para aguas dulces; intervalo que parece brindar protección a la vida de los peces de agua dulce y a los invertebrados que habitan en el fondo; sin embargo, no guarda relación con la productividad y biomasa (Tabla 4) como lo señalara Rodríguez (2008). 


\section{REFERENCIAS BIBLIOGRÁFICAS}

Brower, J., Zar, J., \& von Ende, C. (1990). Field and Laboratory Methods for General Ecology. Wm. C. Brown Publ., 24-51.

Cano, A., La Torre, M. I., León, B., Roque, J., \& Arakaki, M. (1998). Estudio comparativo de la Flora vascular de los Principales Humedales de la Zona Costera del Departamento de Lima. UNMSM - Serie de Divulgación $N^{\circ} 11$, 181-190.

Clarkson, B. R., Sorrell, B. K., Reeves, P. N., Champion, P. D., Partridge, T. R., \& Clarkson, B. D. (2004). Handbook for monitoring wetland condition. Nueva Zelanda: Ministry for the Environment Sustainable Management Fund Project.

Falkowski, P. G., \& Raven, J. A. (2007). Aquatic Photosynthesis. Oxford: Princeton University Press.

Fuam. (2013). Evaluación del biovolumen fitoplanctónico en lagos y humedales interiores de la Comunidad Autónoma del País Vasco y determinación de condiciones de referencia. Madrid: Univ. Autónoma de Madrid.

Gaarder, T., \& Gran, H. H. (1927). Gaarder, T. and Gran, H. H. (1927) Investigation of the production of phytoplankton in the Oslo. Fjord. Rapp. E \& Proc. Verb. Cons. Internet.Explor.Mer. 42:1-48. . Fjord. Rapp. \& Proc. Verb. Cons. Internet. Explor. Mer. , 1-48.

Gil, J., Mineros, E., \& Romero, T. (2013). Estimación de la producción primaria y biomasa del fitoplancton y su relación con algunas condiciones ambientales en la Laguna de Chautengo, Guerrero (Febrero de 2009). El Hombre y su Ambiente, 1-16.
Gras Olivares, D. (1991). El zooplancton del litoral valenciano: su estudio en un medio confinado (Puerto de Valencia) $y$ en mar abierto (Isla de Tabarca). Valencia: Universitat de Valencia.

Jeffrey, S. W., \& Humphrey, G. F. (1975). New Spectrophotometric Equations for Determining Chlorophylls a, b, c1 and c2 in Higher Plants, Algae and Natural Phytoplankton. Biochem. Physiol Pflanzen, 191-194.

Nixon, S. W. (1981). Freshwater inputs and productivity. Fish Wildlife Service, 3157.

Ortega, H., Correa, V., \& Hidalgo, M. (2014). Métodos de colecta, identificación y análisis de comunidades biológicas: plancton, perifiton, bentos (macroinvertebrados) y necton (peces) en aguas continentales del Perú. Lima: Univ. Nac. Mayor de San Marcos.

Parsson, T., Maita, Y., \& Lalli, C. (1984). A manual of chemical and biological methods for seewater analysis. Oxford: Pergamon Press.

Ramsar. (2018 de Abril de 2018). The List of Wetlands of International Importance. Recuperado el 14 de Mayo de 2018, de $r$ a $m$ s $a r$. $\quad$ o $\quad r \quad g$ : https://www.ramsar.org/sites/default/fil es/documents/library/sitelist.pdf

Rodriguez, P. I. (2008). Estructura y producción primaria del fitoplancton y perifiton en un humedal del bajo Paraná. Bs. Aires, Argentina: Univ. de Buenos Aires.

Wilhem, E. (1981). Características Limnológicas de algunos cuerpos de agua del Perú. Lima: Imarpe.

Correo electrónico: milabe@hotmail.com Revisión de Pares:

Recibido: 17 - 04 - 2018

Aceptado: 14 - 06 - 2018 